\title{
MdWRKY46-Enhanced Apple Resistance to Botryosphaeria dothidea by Activating the Expression of MdPBS3.1 in the Salicylic Acid Signaling Pathway
}

\author{
Xian-Yan Zhao, ${ }^{1}$ Chen-Hui Qi, ${ }^{2}$ Han Jiang, ${ }^{1}$ Ming-Shuang Zhong, ${ }^{2}$ Qiang Zhao, ${ }^{2}$ Chun-Xiang You, ${ }^{2}$ \\ Yuan-Yuan $\mathrm{Li}^{2,+}$ and $\mathrm{Yu}-\mathrm{Jin} \mathrm{HaO}^{2, \dagger}$ \\ ${ }^{1}$ State Key Laboratory of Crop Stress Biology for Arid Areas and Shaanxi Key Laboratory of Apple, College of Horticulture, \\ Northwest A\&F University, Yangling 712100, Shaanxi, China \\ ${ }^{2}$ State Key Laboratory of Crop Biology, Shandong Collaborative Innovation Center of Fruit \& Vegetable Quality and Efficient \\ Production, College of Horticulture Science and Engineering, Shandong Agricultural University, Tai-An 271018, Shandong, \\ China
}

Accepted 3 May 2019.

Salicylic acid (SA) is closely related to disease resistance of plants. WRKY transcription factors have been linked to the growth and development of plants, especially under stress conditions. However, the regulatory mechanism of WRKY proteins involved in SA production and disease resistance in apple is not clear. In this study, MdPBS3.1 responded to Botryosphaeria dothidea and enhanced resistance to $B$. dothidea. Electrophoretic mobility shift assays, yeast one-hybrid assays, and chromatin immunoprecipitation and quantitative PCR demonstrated that MdWRKY46 can directly bind to a W-box motif in the promoter of MdPBS3.1. Glucuronidase transactivation and luciferase analysis further showed that MdWRKY46 can activate the expression of MdPBS3.1. Finally, B. dothidea inoculation in transgenic apple calli and fruits revealed that MdWRKY46 improved resistance to $B$. dothidea by the transcriptional activation of MdPBS3.1. Viral vector-based transformation assays indicated that MdWRKY46 elevates SA content and transcription of SA-related genes, including MdPR1, MdPR5, and MdNPR1 in an MdPBS3.1-dependent way. These findings provide new insights into how MdWRKY46 regulates plant resistance to $B$. dothidea through the SA signaling pathway.

\section{Y. Zhao and C. H. Qi contributed equally to the article.}

Sequence data can be found in the GenBank/EMBL data libraries under the following accession numbers: MdPBS3.1 (MD14G1050700); MdPBS3.2 (MD15G1003500); MdWRKY46 (MD07G1146900); and MdACTIN (EB136338).

${ }^{\dagger}$ Corresponding authors: Y. Y. Li; liyy0912@163.com

and Y. J. Hao; haoyujin@sdau.edu.cn

Funding: This work was supported by grants from the National Key Research and Development Program (2018YFD1000200); the National Natural Science Foundation of China (U1706202, 31772275); the Natural Science Fund for Excellent Young Scholars of Shandong Province (ZR2018JL014); and the Ministry of Education of China (IRT15R42).

*The $\boldsymbol{e}$-Xtra logo stands for "electronic extra" and indicates that two supplementary figures and two supplementary tables are published online.

The author(s) declare no conflict of interest.

๑) 2019 The American Phytopathological Society
Keywords: apple, Botryosphaeria dothidea, MdPBS3.1, MdWRKY46, salicylic acid

Salicylic acid (SA), a phenolic derivative, plays important roles in a broad spectrum of pathogenic bacteria ( $\mathrm{Gu}$ et al. 2017; $\mathrm{Lu}$ et al. 2016). Upon infection with a pathogen, plants increase SA production, which affects the redox state of the cell (Chen et al. 1993; Kuźniak et al. 2015; Noctor et al. 2002; Vanacker et al. 2000; Yu et al. 2018). SA can negatively influence the activation of catalase and ascorbate peroxidase or serve as a substrate for ascorbate peroxidase, which may give rise to accumulation of $\mathrm{H}_{2} \mathrm{O}_{2}$, SA-free radicals, and other reactive oxygen species (ROS) (Chen et al. 1993; Durner and Klessig 1995; Herrera-Vásquez et al. 2015; Kvaratskhelia et al. 1997; Xu et al. 2017). Except for its role in triggering redox changes, SA also plays a vital function in maintaining the cellular redox state, probably by inducing the expression of genes involved in the synthesis of antioxidants (Mou et al. 2003). In spite of the critical role of SA in triggering the plant defense response, redundant free SA may be phytotoxic (Kenton et al. 2000; Lee et al. 1995). To maintain normal plant development and growth, plants regulate free SA transformed to SA-O- $\beta$-glucoside (SAG) as the dominant form of SA (Enyedi and Raskin 1993; Jayakannan et al. 2015; Malamy et al. 1992).

The accumulation of SA in plants leads to the expression of pathogenesis-related (PR) proteins and, thus, improves the resistance of plants (Fu and Dong 2013; Klessig et al. 2018; Nawrath and Métraux 1999; Yalpani et al. 1991). When attacked by a pathogen, the accumulation of SA in the Arabidopsis SA induction-deficient 1 (sid1) mutant is blocked (Nawrath and Métraux 1999). Furthermore, sidl mutants are more susceptible to infection by Pseudomonas syringae and Peronospora parasitica, and the expression of $P R I$ is significantly reduced in sid mutants after invasion by these pathogens (Nawrath and Métraux 1999). SID2 (ICS1) also is vital for local and systemic resistance and is responsible for the majority of SA biosynthesis after pathogen infection (Abreu and Munné-Bosch 2009; Wildermuth et al. 2001). Nonexpresser of genes PRl (NPRl) in Arabidopsis plays an important role in SA-mediated disease resistance and is a regulator in systemic-acquired resistance (SAR) (Li et al. 2018). In the absence of NPRl, Arabidopsis cannot respond to SAR-induced treatment, and the expression of $P R$ genes is reduced, making it more susceptible to infection by pathogens (Cao et al. 1997; Mou et al. 2003; Wang et al. 2018). As a result 
of SA accumulation, the redox changes induced during SAR lead to the conformational switch of NPR1, thereby activating $P R$ genes (Mou et al. 2003). A defect in Arabidopsis thaliana phytoalexin deficient 4 (PAD4) affects the expression of the PRI gene and the response to Pseudomonas syringae pv. maculicola ES4326. SA is indispensable for the expression of the $P R 1$ gene. When pad4 is inoculated with $P$. syringae pv. maculicola ES4326, SA accumulation and $P R 1$ gene expression is reduced, which causes defects in the defense response (Zhou et al. 1998). The enhanced disease susceptibility 1 (EDS1)/PAD4 complex is capable of inducing expression of SA response genes and resistance to pathogens (Cui et al. 2016). P. syringae pv. tomato DC3000 treatment can induce the expression of SA-related genes $(P R 1, P R 2$, and $P R 5)$ and bring about an increase in production of SA (Jia et al. 2018).

AVR PPHB SUSCEPTIBLE 3 (PBS3), a member of the GH3 acyl adenylase family, functions upstream of SA synthesis, either in a regulatory capacity, which may include an amplification loop, or in SA biosynthesis (Nobuta et al. 2007). The pbs3 mutant in Arabidopsis affects SAG accumulation and SA-dependent gene expression, and the pbs3 mutant shows a deficiency in resistance upon invasion by pathogens. PBS3 plays an important role in regulating SA-dependent resistance responses (Nobuta et al. 2007). The HopW1-1 of P. syringae pv. maculicola ES4326 effector in $P$. syringae can target $P B S 3$ and alter the SA signaling pathway (Lee et al. 2008). PBS3 acts upstream of the SA signal to regulate pathogen resistance (Wang et al. 2011), but the transcriptional regulation of $P B S 3$ in plants is not clear.

In a complex environment, plants are subjected to a variety of biological stresses, among which transcription factors play a crucial role in plant immunity induced by biological stresses (Pandey and Somssich 2009; Sarris et al. 2015). In strawberries, for instance, the expression of $F v W R K Y 42$ and $P R l$ is induced by SA and increases plant resistance to powdery mildew (Wei et al. 2018). Arabidopsis expression of AtWRKY46 is upregulated by $P$. syringae or SA, and AtWRKY46 can coordinate with WRKY70 and WRKY53 to increase resistance to $P$. syringae (Hu et al. 2012). In addition, several WRKY transcription factors act as negative regulators of biotic resistance. In Arabidopsis, analyses of mutants has revealed that the loss of WRKY11 function increases resistance to $P$. syringae pv. tomato strains and that resistance is further enhanced in wrkyl1 wrkyl7 doublemutant plants (Journot-Catalino et al. 2006). Arabidopsis WRKY18/40 can negatively regulate the expression of EDS1 and $P A D 4$ and promote powdery mildew infection (Pandey et al. 2010). In Capsicum annuum, CaWRKY58 negatively regulates plant resistance to Ralstonia solanacearum, and plants with decreased CaWRKY58 expression display increased resistance to $R$. solanacearum (Wang et al. 2013). These findings indicate that WRKY proteins play crucial roles in plant immune responses.

Botryosphaeria dothidea is a model strain of Botryosphaeria spp. (Marsberg et al. 2017). On apple, B. dothidea mainly harms the branches and fruits. It also adversely affects tree growth, releasing spores that infect the fruit, which results in rot and in the death of branches and young trees. The expression of $M d P R-4$ in apples can be induced by SA, which mediates the resistance of apples to $B$. dothidea (Bai et al. 2013). After the inhibition of syntaxin-related 121 (SYP121) expression in apples, resistance to $B$. dothidea is improved (He et al. 2018). However, the regulatory mechanism of WRKY transcription factors in relation to $B$. dothidea infection is not well studied.

In this study, we found that MdPBS3.1 expression can be induced by $B$. dothidea, and resistance to $B$. dothidea is improved by MdPBS3.1 overexpression. The WRKY transcription factor MdWRKY46 activated MdPBS3.1 expression by directly binding to the W-box motif in the promoter of MdPBS3.1, which positively regulated the resistance to $B$. dothidea through the
SA-mediated pathway. These findings provide important insights into the function and the novel regulatory mechanism of MdWRKY46 in mediating resistance to $B$. dothidea through the SA signaling pathway in apples, which may help to defend apples from infection by $B$. dothidea.

\section{RESULTS}

\section{MdPBS3.1 response to $B$. dothidea.}

In plants, pathogens are able to induce SA accumulation (Nawrath and Métraux 1999). PBS3 plays an important role in the production of SA in plants (Nobuta et al. 2007). B. dothidea is an important pathogen causing disease of apple branches and fruits, and it is considered a major threat to the fruit-producing industry. To investigate the relationship between $B$. dothidea and $P B S 3$ in apples, a BLAST search against the apple genome database was conducted using Arabidopsis AtPBS3 (At5g13320) as the query. As a result, 21 PBS3-like genes were found, and a sequence alignment of 21 probable PBS3 homologs in apple with Arabidopsis PBS3 was made (Supplementary Fig. S1). Two proteins, MdPBS3.1 (MDP0000257967) and MdPBS3.2 (MDP0000716496), were selected according to their high protein similarity to AtPBS3. On the basis of a new apple genome database, MdPBS3.1 (MDP0000257967) is MdPBS3.1 (MD14G1050700), and MdPBS3.2 (MDP0000716496) is MdPBS3.1 (MD15G1003500).

The expression levels of MdPBS3.1 and MdBS3.2 were examined after infection of 'Fuji' apples with $B$. dothidea. Compared with the control, the $M d P B S 3.1$ expression level was significantly increased 1 and 2 days after inoculation with $B$. dothidea. Although the $M d P B S 3.2$ expression level also increased 1 and 2 days after inoculation with $B$. dothidea, the extent of the increase was not comparable to that of MdPBS3.1 (Fig. 1A); therefore, the decision was made to focus on MdPBS3.1 in this study.

To investigate the function of $M d P B S 3.1$, the National Center for Biotechnology Information (NCBI) conserved-domains online software was used to analyze the conserved domain of the MdPBS3.1 protein. The relationship between apple MdPBS3.1 and PBS3.1 from other species also was analyzed. The results showed that the PBS3.1 from 13 species could be divided into three classes. Class I contained PBS3.1 from tobacco (Nicotiana sylvestris) and pear (Pyrus $\times$ bretschneideri), whereas class III contained PBS3.1 from astragalus (Herrania umbratica), rose (Rosa chinensis), cassava (Manihot esculenta), cacao (Theobroma cacao), pea (Parasponia andersonii), and dungarunga (Trema orientale). MdPBS3.1 belonged to class II, which contained PBS3.1 from loquat (Eriobotrya japonica), club (Prunus mume), cherry (Prunus avium), and peach (Prunus persica), which all belong to the Rosaceae family. Apple MdPBS3.1 showed the strongest relationship to loquat PBS3.1, suggesting they may have similar functions (Fig. 1B). In addition, MdPBS3.1 primarily contained an adenosine monophosphate (AMP)-binding site (Fig. 1C), indicating that MdPBS3.1 belongs to the AMP-binding superfamily.

\section{MdPBS3.1 confers increased resistance to $B$. dothidea in apple calli.}

To investigate the role of $M d P B S 3.1$ in resistance to B. dothidea, MdPBS3.1-overexpressing and MdPBS3.1-antisense apple calli were obtained (Supplementary Fig. S2A and B). After inoculation with $B$. dothidea, MdPBS3.1 overexpression reduced the diameter of the disease spot in apple calli compared with the wild type (WT). (Fig. 2A and B). This result was further confirmed by quantitative real-time PCR (qRTPCR) detection of the expression of pathogen-associated genes. The expression of $M d P R 1, M d P R 5$, and $M d N P R 1$ was significantly induced in the $M d P B S 3.1$-overexpressing calli 
(Fig. 2C). These results further demonstrated that MdPBS3.1 can increase resistance to $B$. dothidea through the SA pathway.

The W-box 2 in the promoter sequence of MdPBS3.1 is crucial for its response to $B$. dothidea.

Although MdPBS3.1 confers apple calli with increased resistance to $B$. dothidea, the regulatory mechanism of MdPBS3.1 in response to $B$. dothidea is unclear. To further investigate the activation of MdPBS3.1 by B. dothidea, the proMdPBS3.1-GUS vector was constructed and transiently transformed into 'Royal Gala' apple seedlings by vacuum infiltration. After the transformed plants were treated with $B$. dothidea for $6 \mathrm{~h}$, glucuronidase (GUS) was intensely stained. Compared with $B$. dothidea treatment for $6 \mathrm{~h}$, the apple seedlings could still be stained by
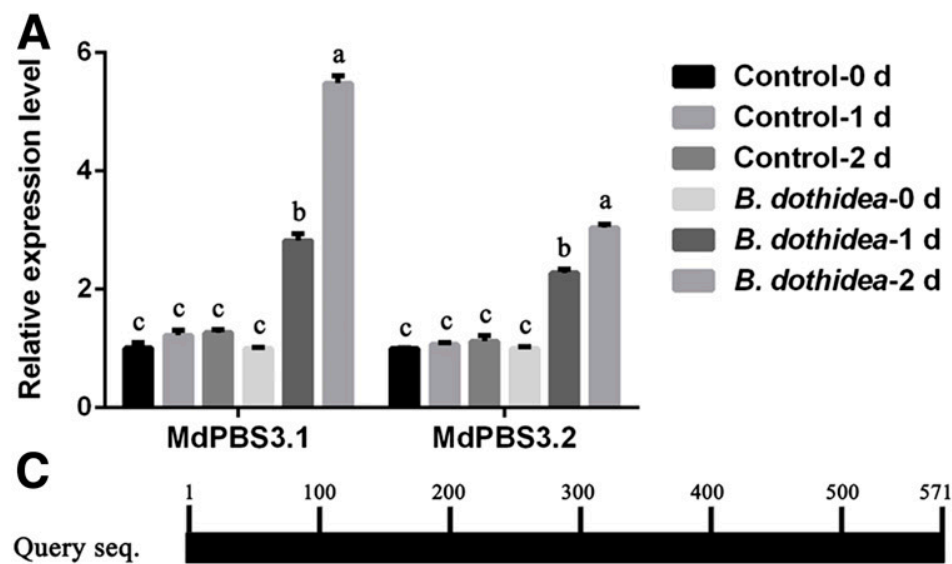

B

Query seq.

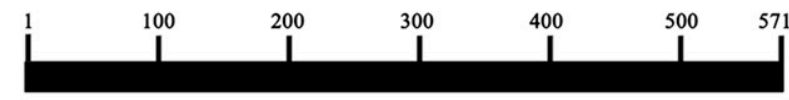

Superfamilies
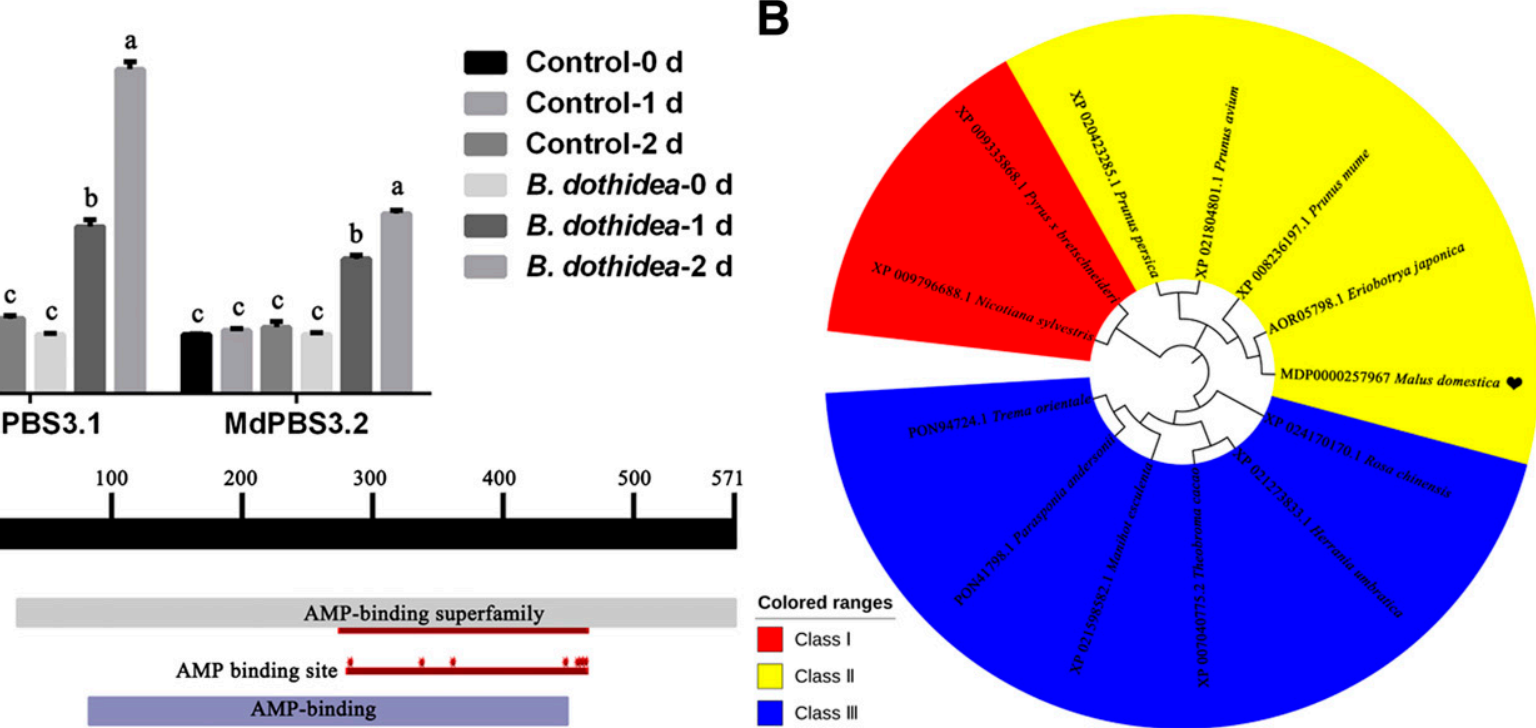

Fig. 1. The expression of MdPBS3 genes induced by Botryosphaeria dothidea. A, Expression of MdPBS3.1 and MdPBS3.2 in 'Fuji' apples treated with or without B. dothidea for 0 days, 1 day, and 2 days. Untreated apples were used as the control. B, Phylogenetic tree between apple MdPBS3.1 and PBS3.1 from other species, including Malus domestica, Eriobotrya japonica, Pyrus bretschneideri, Prunus avium, Prunus persica, Prunus mume, Rosa chinensis, Theobroma cacao, Trema orientale, Parasponia andersonii, Manihot esculenta, Herrania umbratica, and Nicotiana sylvestris. C, Conserved domain of the MdPBS3.1 protein.

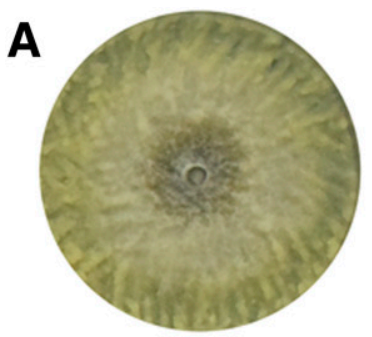

WT

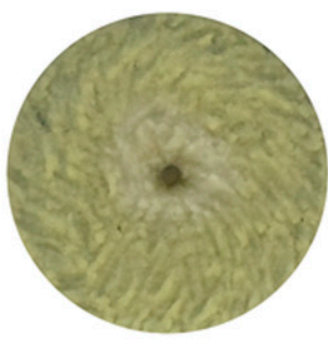

MdPBS3.1-OE-4

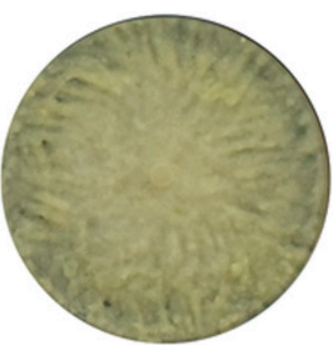

MdPBS3.1-Anti-2

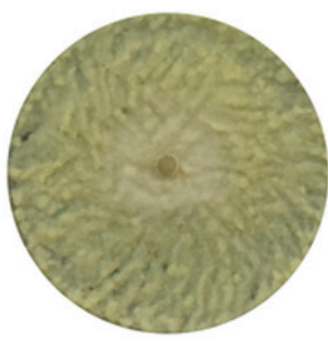

MdWRKY46-OE-3

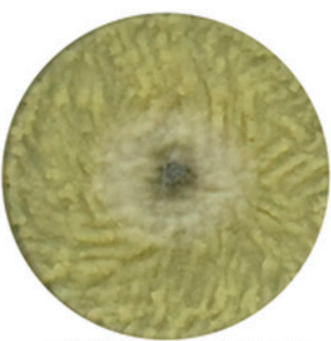

MdWRKY46-OE-3/

MdPBS3.1-Anti-4
B

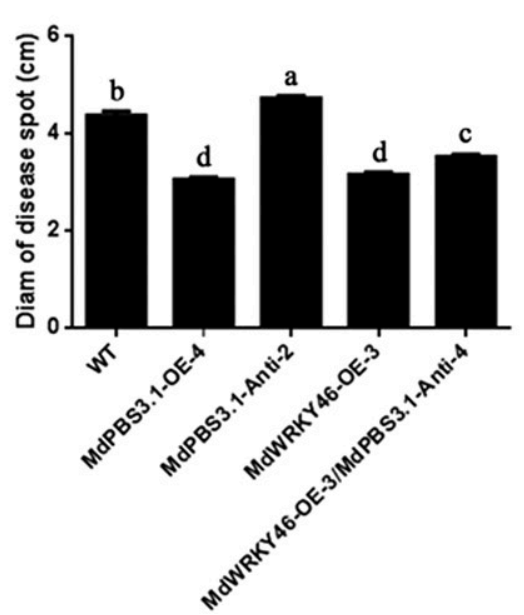

C

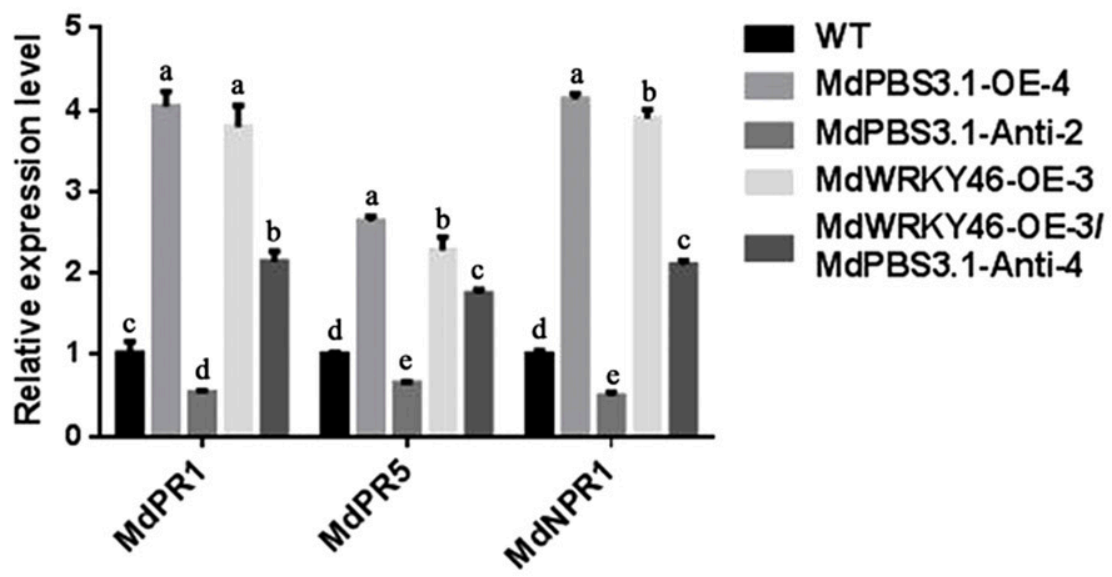

Fig. 2. Enhanced resistance to Botryosphaeria dothidea of MdWRKY46 in an MdPBS3.1-dependent manner in apple calli. A, Phenotype of wild type, MdPBS3.1 overexpressing, MdPBS3.1 antisense apple calli, MdWRKY46 overexpressing, and MdWRKY46 overexpressing and MdPBS3.1 antisense doubletransgenic apple calli inoculated with $B$. dothidea. B, Diameters of disease spots of different apple calli in A. C, Expression levels of MdPR1, MdPR5, and MdNPR1 in different types of apple calli. 
GUS after $12 \mathrm{~h}$ and $24 \mathrm{~h}$ of treatment, but the color became much lighter, suggesting low GUS activity (Fig. 3A). The results of experiments that investigated $G U S$ expression yielded consistent results (Fig. 3D), indicating that $M d P B S 3.1$ was induced by B. dothidea.

The promoter of MdPBS3.1 was analyzed, and many cis-elements, such as MBS, W-box, AREB, and others, were found (Supplementary Table S2). Studies have reported that many WRKY transcription factors are involved in plant resistance in the SA pathway, and W-box (TTGACT/C) is considered the WRKY transcription factor-binding site. In this study, the focus was on the two W-box motifs in the MdPBS3.1 promoter: proMdPBS3.1GUS ( $\mathrm{ml}$ ) was constructed with the first W-box (W-box1) mutated to TACGTC, and proMdPBS3.1-GUS $(\mathrm{m} 2)$ was constructed with the second W-box (W-box2) mutated to TACGTC. Thereafter, the two vectors were transformed to the 'Royal Gala' apple seedlings and treated with $B$. dothidea. GUS staining and proMdPBS3.1GUS (m2) activity in apple seedlings were reduced compared with the nonmutant MdPBS3.1 promoter. However, proMdPBS3.1GUS ( $m 1$ ) GUS analysis showed no apparent changes (Fig. 3B

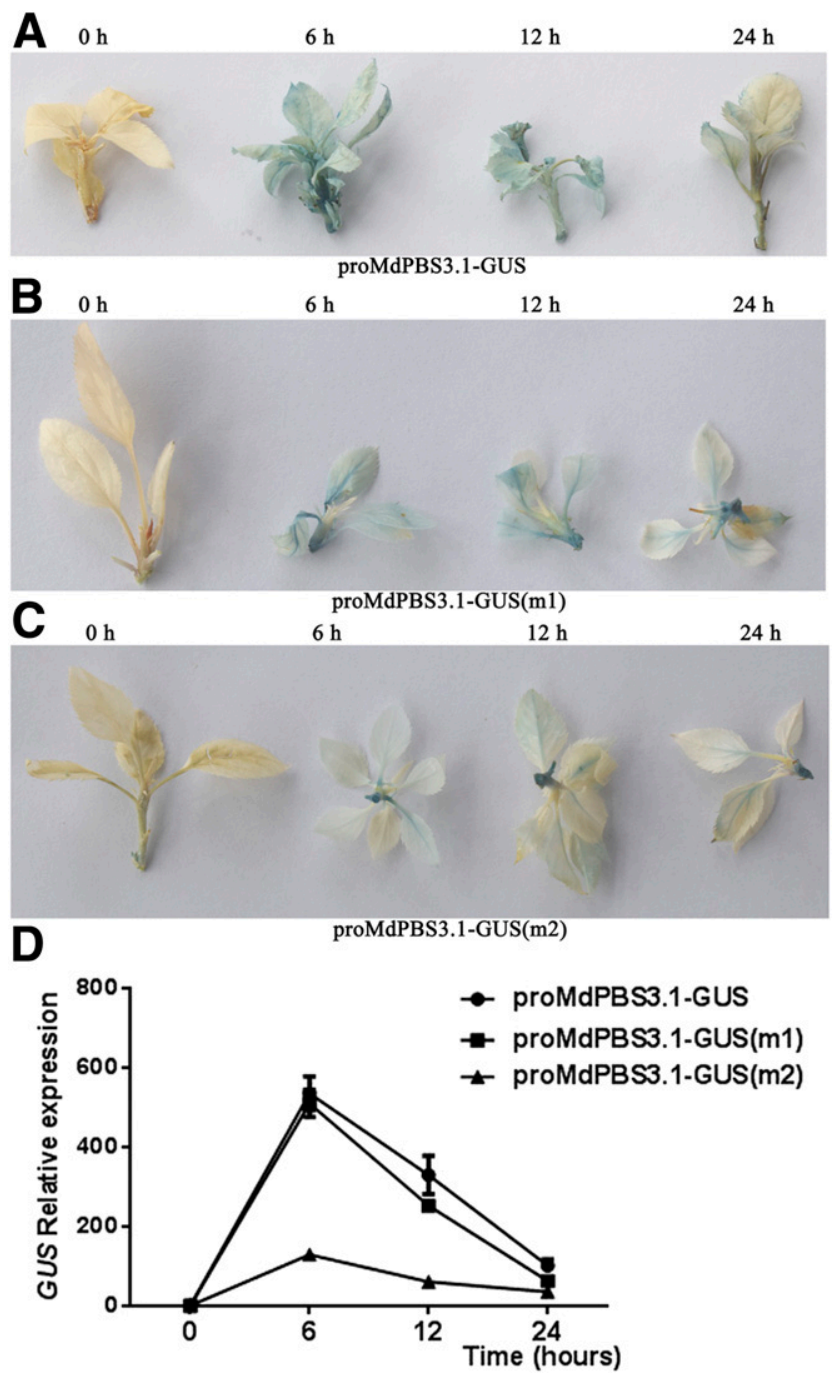

Fig. 3. Glucuronidase (GUS) staining of the MdPBS3.1 promoter after its response to Botryosphaeria dothidea. A, Apple seedlings transferred to the MdPBS3.1 promoter (proMdPBS3.1-GUS) and $\mathbf{B}$ and $\mathbf{C}$, apple seedlings transferred to the mutated MdPBS3.1 promoter (proMdPBS3.1-GUS ( $\mathrm{ml}$ or $m 2)$ ) were analyzed by GUS staining. D, GUS relative expression after treating with $B$. dothidea for $0 \mathrm{~h}, 6 \mathrm{~h}, 12 \mathrm{~h}$, and $24 \mathrm{~h}$, respectively. proMdPBS3.1-GUS ( $m 1$ or $m 2$ ) represents the mutant promoter of $M d P B S 3.1$ in which W-box1 or W-box2 (TTGACA) was mutant to TACGTC, respectively. and D). These results indicate that the W-box 2 of the MdPBS3.1 promoter is crucial for response to $B$. dothidea.

\section{MdWRKY46 binds the W-Box2}

in the promoter sequence of MdPBS3.1.

To identify the potential WRKY transcription factors that regulate the expression of $M d P B S 3.1$, a yeast single-hybrid screen library was prepared using the MdPBS3.1 promoter fragment, with the approximately 739-bp W-box2 as the bait. One positive clone encoding probable WRKY transcription factor 46 (MdWRKY46, MD07G1146900) was identified.

To examine the interaction of MdWRKY46 and the promoter of $M d P B S 3.1$, an electrophoretic mobility shift assay (EMSA) was carried out to detect binding of MdWRKY46 to the promoter region of MdPBS3.1. The results indicate MdWRKY46 was bound directly to the promoter $\mathrm{W}$-box 2 of $M d P B S 3.1$ in vitro (Fig. 4A and B). As shown in Figure 4B, after the W-box2 in the MdPBS3.1 promoter was mutated to TACGTC, binding disappeared in the EMSA (Fig. 4B), indicating that the binding was specific. Thereafter, a yeast one-hybrid $(\mathrm{Y} 1 \mathrm{H})$ assay was carried out to confirm the binding of MdWRKY46 to the promoter of $M d P B S 3.1$. The results showed that MdWRKY46-AD and proMdPBS3.1-box2-pHIS2-cotransformed yeast cells were able to grow in the -Trp/-Leu/-His medium supplemented with $20 \mathrm{mM}$ 3-amino-1,2,4-triazole (3-AT), but the negative control cotransformed with $A D$ and proMdPBS3.1-box2-pHIS2 failed to grow (Fig. 4C). These findings indicate that MdWRKY46 specifically binds to the $\mathrm{W}$-box 2 of the $M d P B S 3.1$ promoter in vivo.

To further demonstrate that MdWRKY46 can bind the promoter of MdPBS3.1, MdWRKY46 fused with GFP-tagged transgenic calli was obtained (Fig. 4D). Chromatin immunoprecipitation and quantitative PCR (ChIP-qPCR) assays were performed using MdWRKY46 transgenic calli and GFP transgenic calli. The results show that the MdPBS3.1 promoter fragments were enriched with MdWRKY46 transgenic calli (Fig. 4E), suggesting that MdWRKY46 can directly interact with the W-box 2 motif in the promoter region of MdPBS3.1.

\section{Transcriptional activation of MdPBS3.1 by MdWRKY46.}

The aforementioned results indicate that MdWRKY46 was able to bind to the promoter of MdPBS3.1. To further examine whether the transcriptional activity of $M d P B S 3.1$ was induced or suppressed by MdWRKY46, a GUS reporter gene was fused downstream of the 739-bp MdPBS3.1 promoter and defined as proMdPBS3.1-box2-GUS. Additionally, the open reading frame of MdWRKY46 was inserted into the pGreenII 62-SK vector under the control of the CaMV 35S promoter. Thereafter, the recombinant MdWRKY46-pGreenII 62-SK and proMdPBS3.1-box2-GUS plasmids were used in a GUS transactivation assay using 'Orin' apple calli. GUS activity assays and western blotting analysis also were performed. The results showed that GUS staining of MdWRKY46-pGreenII 62-SK and proMdPBS3.1-box2-GUS calli produced a stronger color than in the other combinations, including pGreenII 62-SK + 1300-GUS, MdWRKY46-pGreenII 62-SK + 1300-GUS, and pGreenII 62-SK + proMdPBS3.1-box2GUS (Fig. 5A). GUS activity also was higher in western blotting, indicating that MdWRKY46 bound to the promoter of MdPBS3.1 to activate its transcription (Fig. 5A and B).

Subsequently, a firefly luciferase (LUC) complementation imaging assay was carried out to verify the activation of MdWRKY6 to $M d P B S 3.1$ in vivo. A $L U C$ gene was fused downstream of the $M d P B S 3.1$ promoter and defined as proMdPBS3.1-box2pGreenII 0800-LUC. The luminescence intensity was significantly increased compared with the controls when MdWRKY46pGreenII 62-SK and proMdPBS3.1-box2-pGreenII 0800-LUC were coinjected into tobacco (Fig. 5C), further demonstrating that MdWRKY46 increased MdPBS3.1 expression. 
Thereafter, proMdPBS3.1-box2-GUS was transformed into apple calli to generate proMdPBS3.1-box2-GUS-overexpressing apple calli. MdWRKY46 was then transiently transformed into proMdPBS3.1-box2-GUS and 1300-GUS transgenic calli, respectively. GUS activity in the two transgenic calli was compared, which revealed that double-transgenic calli contained MdWRKY46 and that proMdPBS3.1-box2-GUS promoted the transcriptional activity of GUS compared with the 1300-GUS-overexpressing calli (Fig. 5D and E). In addition, MdPBS3.1 expression was significantly increased in MdWRKY46 transgenic calli (Fig. 5F). These results indicated that MdWRKY46 can transcriptionally activate the expression of MdPBS3.1 by binding to its promoter.

MdWRKY46 enhances resistance to $B$. dothidea in an MdPBS3.1-dependent manner in apple.

To confirm the biological function of $M d W R K Y 46$, the transcriptional level of $M d W R K Y 46$ after SA treatment was examined, and SA was found to significantly induce MdWRKY46 expression, indicating its potential function in the SA signaling pathway. $B$. dothidea mainly affects the branches and fruits of apples. To examine whether MdWRKY46 regulated MdPBS3.1modulated resistance to $B$. dothidea in apple fruits, $B$. dothidea was inoculated into VIGS-injected apple peel. Both pIR$M d P B S 3.1$ and pIR-MdWRKY46 increased resistance to $B$. dothidea compared with the control $(p I R)$. In contrast, $T R V$ $M d P B S 3.1$ reduced resistance to $B$. dothidea compared with the control (TRV). The results of coinjecting $p I R-M d W R K Y 46$ and $T R V-M d P B S 3.1$ were similar to those of $T R V-M d P B S 3.1$ (Fig. $6 \mathrm{~A}$ and $\mathrm{B}$ ), indicating the positive roles of MdWRKY46 and $M d P B S 3.1$ in B. dothidea resistance. Next, the free SA and SAG levels 2 days after injection were measured. When MdPBS3.1 or MdWRKY46 were overexpressed, the free SA and SAG levels increased compared with the control. When the expression of MdPBS3.1 was inhibited, however, the free SA and SAG levels

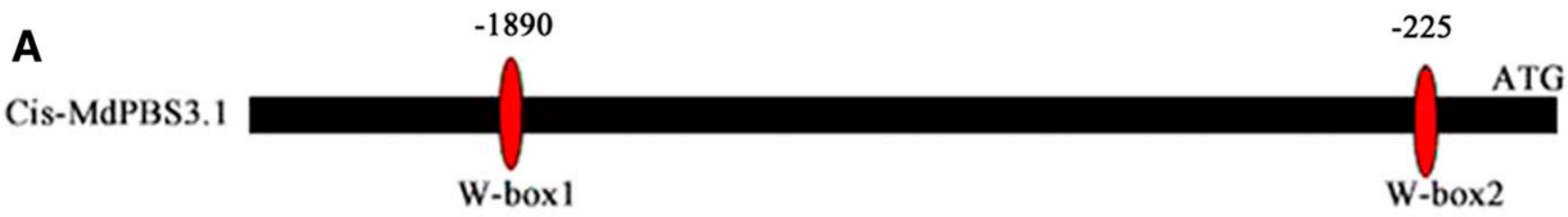

B

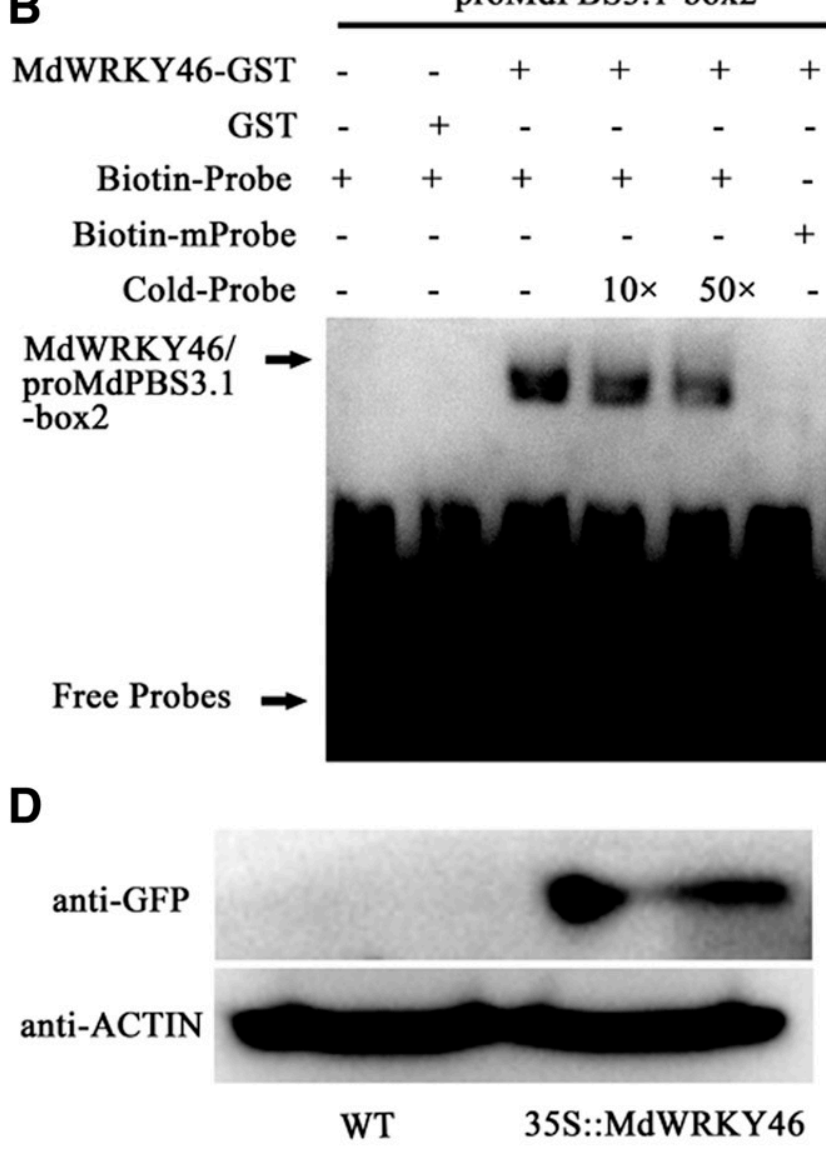

C

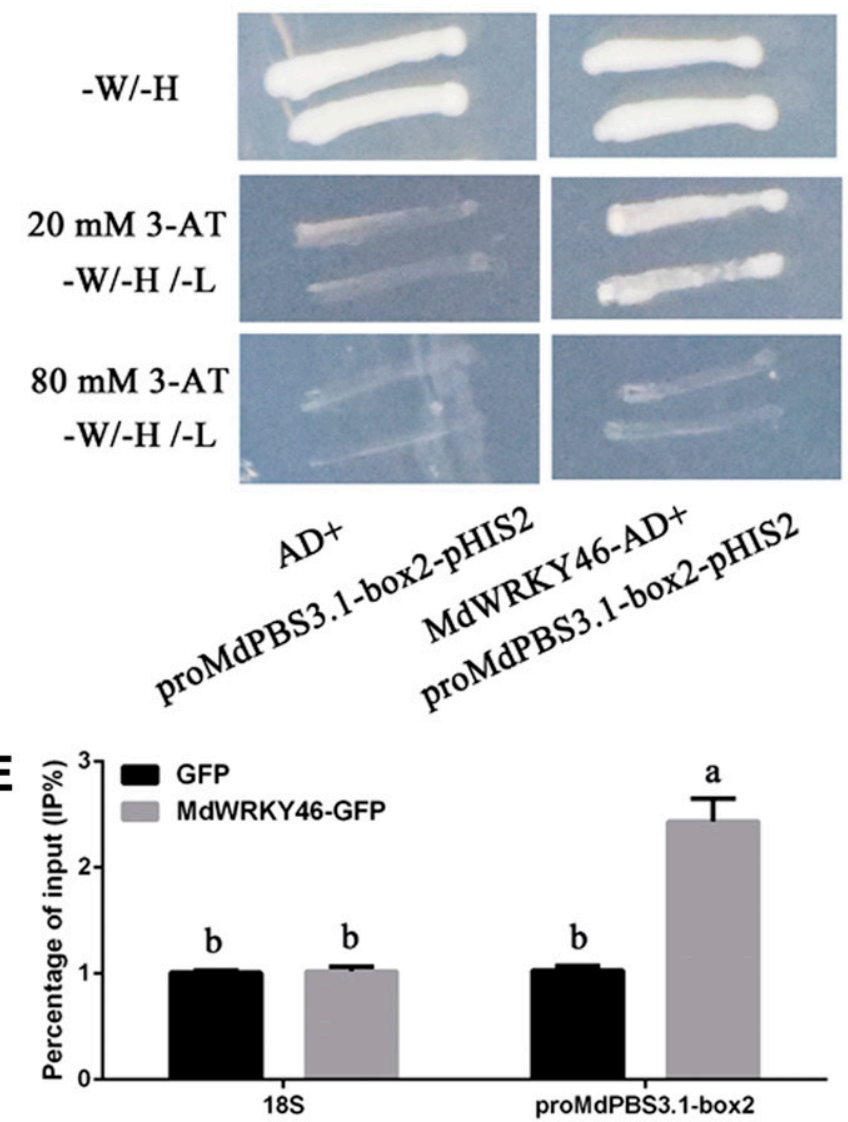

Fig. 4. The binding of MdWRKY46 on the MdPBS3.1 promoter. A, Schematic map of W-box in the promoter of MdPBS3.1. B, Electrophoretic mobility shift assays were used to detect binding of MdWRKY46 to the MdPBS3.1 promoter. Each biotin-labeled DNA probe was incubated with MdWRKY46-GST protein. The mutated probe of $\mathrm{P}$ had a mutation in the W-box (TTGACA was replaced by TACGTC). C, Yeast one-hybrid assays were used to detect the transcriptional activation of MdPBS3.1 by MdWRKY46. The constructs were cotransformed into yeast Y187. Yeast was cultured on a culture medium devoid of tryptophan and histidine (SD/-W/-H) and then screened for the lack of tryptophan, leucine, and histidine (SD/-W/-L/-H) with 20 mM and 80 mM 3-AT (3-amino-1,2,4triazole). D, MdWRKY46 protein expression using an anti-GFP antibody in MdWRKY46 overexpressed calli and wild-type calli. Anti-ACTIN was used as the loading control. E, Chromatin immunoprecipitation and quantitative PCR assays were performed to detect enriched DNA fragments of MdWRKY46overexpressing calli (MdWRKY46-GFP) and calli transformed with an empty vector $(G F P)$. MdACTIN served as the control. 
decreased (Fig. 6C and D). These results indicate that SA may be involved in resistance to $B$. dothidea through the MdWRKY46mediated MdPBS3.1 pathway.

In addition, the expression of $P R$ genes $(M d P R 1, M d P R 5$, $M d N P R 1)$ after injection was examined. Compared with the control, the expression of $P R$ genes was significantly upregulated after $M d W R K Y 46$ or $M d P B S 3.1$ overexpression, whereas expression of $M d P B S 3.1$ was inhibited or expression of $P R$ genes decreased after coinjection of TRV-MdPBS3.1 and $p I R$ MdWRKY46 (Fig. 6D).

The function of MdWRKY46 and MdPBS3.1 in resistance to $B$. dothidea was further confirmed in transgenic apple calli. $M d W R K Y 46$-overexpressing, MdWRKY46-overexpressing, and $M d P B S 3.1$-antisense double-transgenic apple calli were obtained. After inoculation with $B$. dothidea, MdWRKY46 overexpression reduced the diameter of the disease spot compared with the WT, and when expression of MdPBS3.1 was inhibited, the diameter of the disease spot increased. The disease spot was also larger in double-transgenic calli than in the WT (Fig. 2A and C). These results were further confirmed by qRT-PCR used to detect expression of $P R$ genes. The expression of $M d P R 1, M d P R 5$, and $M d N P R 1$ was significantly increased in MdPBS3.1-overexpressing and MdWRKY46-overexpressing calli (Fig. 2D). These results indicated that MdWRKY46 and MdPBS3.1 are able to positively regulate resistance to $B$. dothidea. They also demonstrated that MdWRKY46 may improve resistance to $B$. dothidea through an MdPBS3.1-dependent pathway.

\section{DISCUSSION}

In plants, specific disease resistance $(\mathrm{R})$ proteins identify pathogens and enhance resistance to disease (Jones and Dangl 2006; Wang et al. 2015). R proteins bring about a complex series of defense responses, such as the strengthening of the cell wall, bursts of ROS, and accumulation of antimicrobial proteins, known as PR proteins (Hammond-Kosack and Jones 1996; Lehmann et al. 2015). SA is considered a key second messenger involved in the induction of PR proteins and the expansion of the oxidative burst (Lu et al. 2016; Ryals et al. 1996; Shirasu et al. 1997; Yalpani et al. 1991).

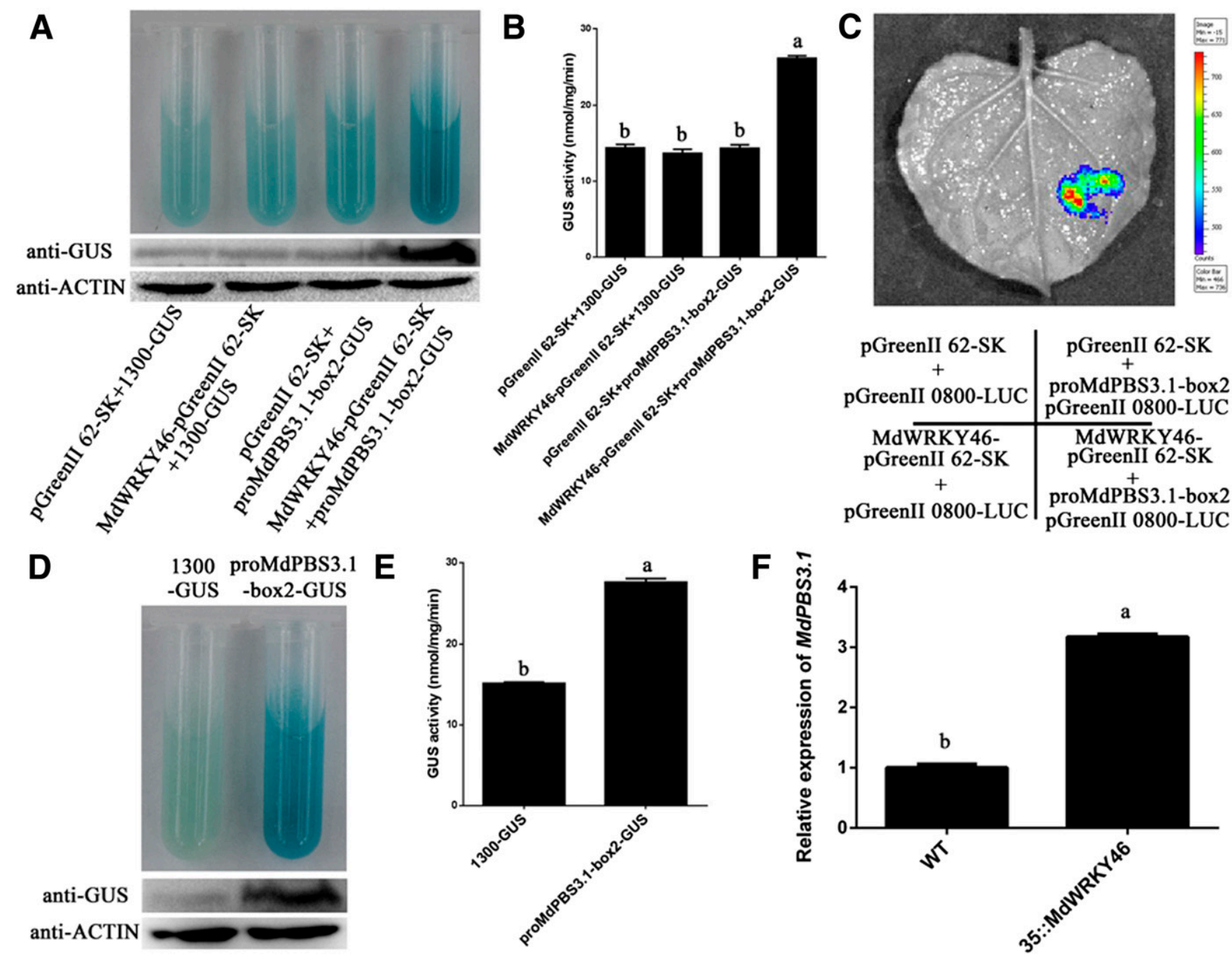

Fig. 5. Transcriptional activation of MdPBS3.1 by MdWRKY46. A and B, pGreenII 62-SK + 1300-GUS, MdWRKY46-pGreenII 62-SK + 1300-GUS, pGreenII 62-SK + proMdPBS3.1-box2-GUS, and MdWRKY46-pGreenII 62-SK + proMdPBS3.1-box2-GUS were transformed into apple calli for glucuronidase (GUS) staining, immunoblotting (A), and GUS activity (B). C, Dual luciferase assays in tobacco leaves. proMdPBS3.1-box2-pGreenII 0800-LUC + MdWRKY46-pGreenII 62-SK was transiently transformed into tobacco leaves to detect luminescence, and pGreenII 0800-LUC + pGreenII 62-SK, pGreenII 0800-LUC + MdWRKY46-pGreenII 62-SK, and proMdPBS3.1-box2-pGreenII 0800-LUC + pGreenII 62-SK were used as the controls. D and E, MdWRKY46-GFP was transiently transformed into proMdPBS3.1-GUS transgenic calli or 1300-GUS transgenic calli to detect GUS staining (D) and GUS activity (E). F, Expression of MdPBS3.1 in wild-type (WT) and MdWRKY46 transgenic calli. WT represents wild-type 'Orin' apple calli. All experiments were repeated at least three times, and the same results were obtained each time. 1300-GUS: empty gus reporter. 
The responses of MdPBS3.1 to $\mathrm{B}$. dothidea.

SA plays important roles in plant disease resistance ( $\mathrm{Lu}$ et al. 2016; Shirasu et al. 1997; White 1979). A major portion of SA produced in plants is converted into SAG, which is the dominant form of SA (Barman et al. 2016; Enyedi and Raskin 1993; Malamy et al. 1992). PBS3 belongs to the GH3 family of acyl adenylases/thioester-forming enzymes that contribute to the accumulation of SAG (Nobuta et al. 2007; Staswick et al. 2002). A pbs3-1 mutant has been shown to decrease the resistance to P. syringae (avrRpt2) infection (Nobuta et al. 2007). PBS3 has an AMP-binding motif that is necessary for adenylation (Nobuta et al. 2007). AMP-binding and phylogenetic tree analysis of MdPBS3.1 revealed that the function of MdPBS3.1 was conserved among different plant species (Fig. 1). Based on the results, MdPBS3.1 could be induced by $B$. dothidea (Fig. 1), suggesting that MdPBS3.1 may be associated with resistance to $B$. dothidea. The response of the MdPBS3.1 promoter to $B$. dothidea (Fig. 3) further implied that MdPBS3.1 plays a vital role in the disease resistance of apple.

\section{The increased SA content influenced} by MdWRKY46 and MdPBS3.1.

The GH3 family was first found in soybean plants (Hagen et al. 1984). In Arabidopsis, 19 GH3 genes have been identified and divided into three distinct groups based on protein analysis (Staswick et al. 2005; Westfall et al. 2010). PBS3 belongs to group III of the $\mathrm{GH} 3$ proteins. A benzoyl-amino acid synthetase (AtPBS3) is involve in the SA pathway (Nobuta et al. 2007; Okrent et al. 2009). In the process of plant immunity, PBS3 protects EDS1 from proteasome-mediated degradation (Chang et al. 2019). MdPBS3.1 can enhance resistance to $B$. dothidea and the content of free SA and SAG (Fig. 2). Under normal circumstances, however, free SA remains at its basal level, at which it does not affect the growth and development of plants (Hao et al. 2018). When inoculated with $B$. dothidea, SAG may convert to SA to activate resistance. During the conversion of SAG and free SA, MdPBS3.1 plays an indispensable role. In addition, the weak response of the promoter of MdPBS3.1 to $B$. dothidea, in which the $\mathrm{W}$-box 1 motif was mutated, indicates there may exist vital WRKY transcription factors that are related to resistance to $B$. dothidea.

A growing body of research indicates that WRKY proteins are involved in responses to biotic stress in a manner that involves transcriptional activation or inhibition (Dong et al. 2003; Kim et al. 2006; Li et al. 2006). In tobacco, NtWRKY12 binds to the $P R l$ promoter and activates the transcription of $P R l$ by SA and bacterial elicitors (van Verk et al. 2008). Arabidopsis
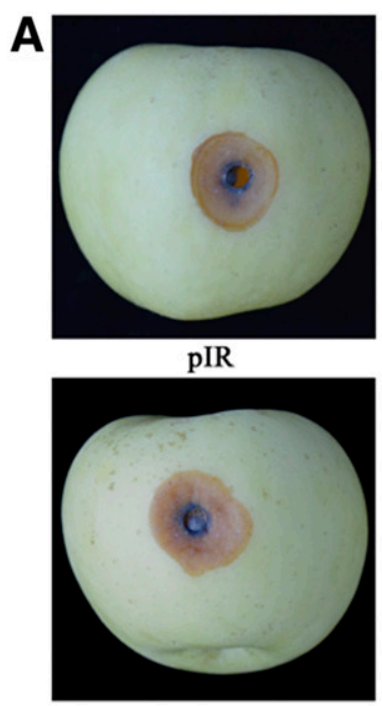

TRV

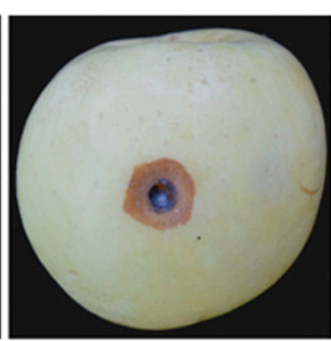

pIR-MdPBS3.1

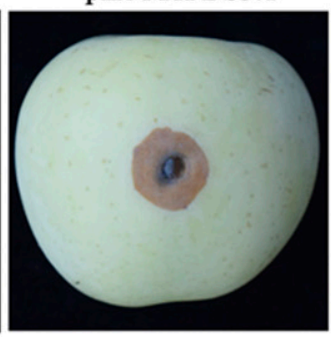

pIR-MdWRKY46

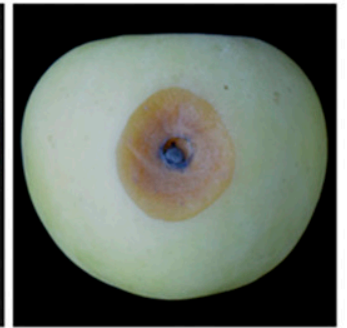

TRV-MdPBS3.1

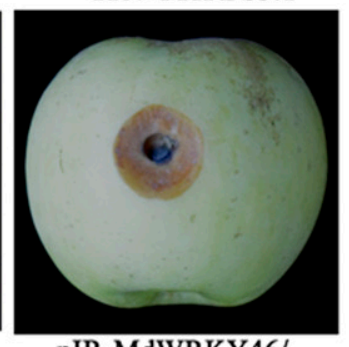

pIR-MdWRKY46/
B

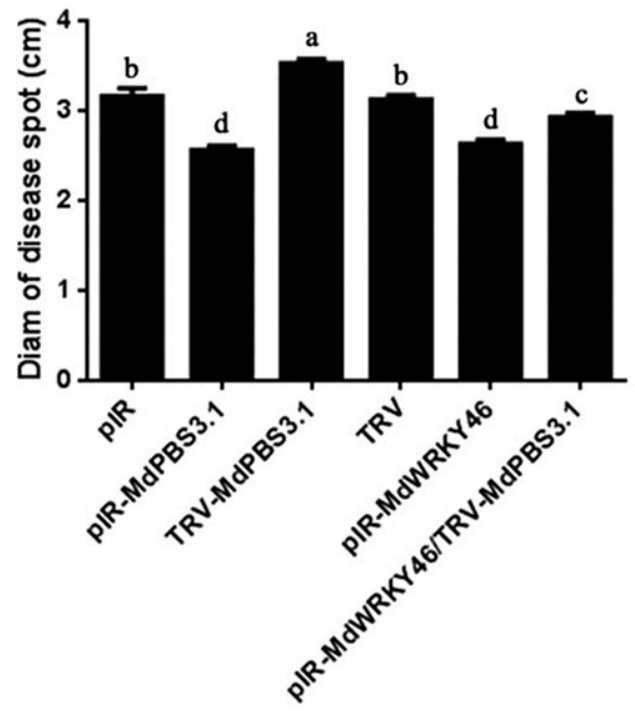

C

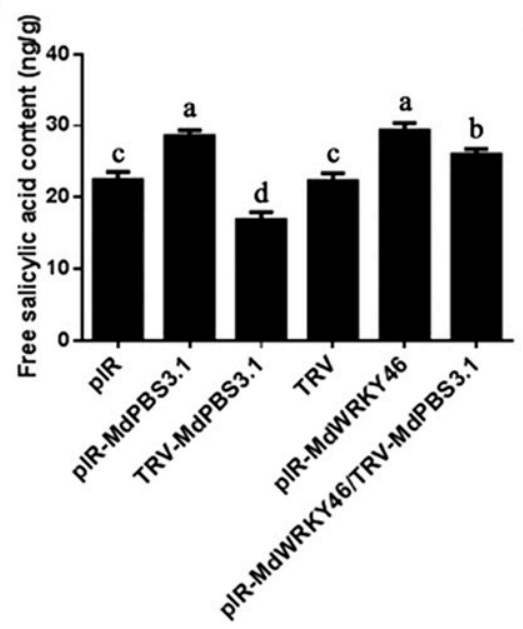

D

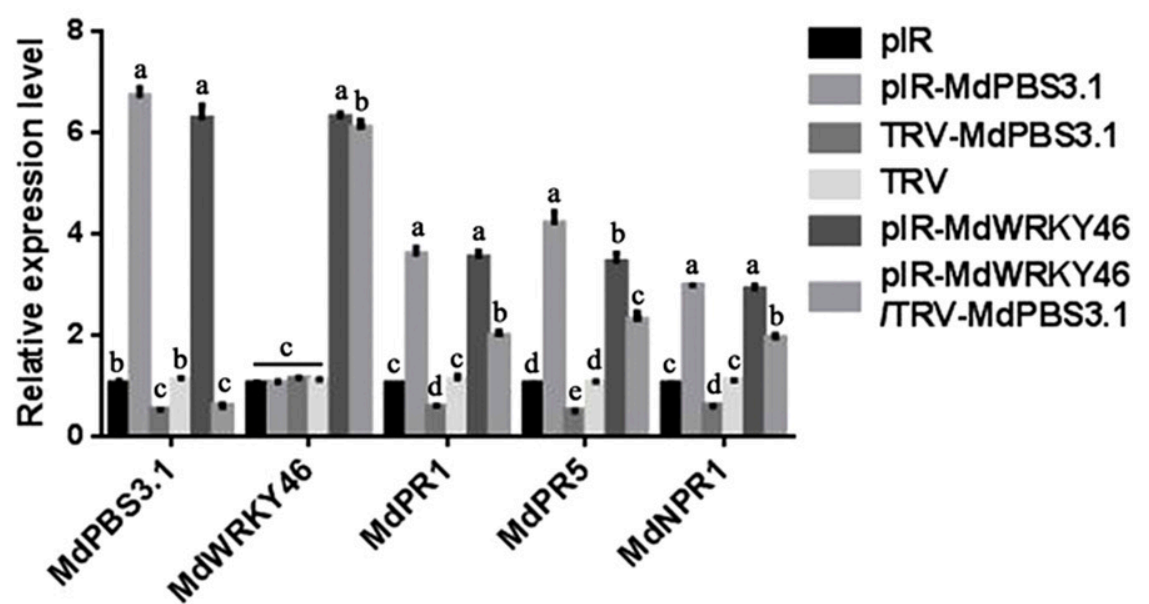

Fig. 6. Increased resistance of MdWRKY46 against Botryosphaeria dothidea in an MdPBS3.1-dependent manner in apple. A, pIR-MdWRKY46, TRVMdPBS3.1, pIR-MdPBS3.1, pIR-MdWRKY46, and TRV-MdPBS3.1 were transiently transformed into 'Fuji' apple, and phenotypic changes were observed after inoculation with $B$. dothidea. B, Lesion diameter; $\mathbf{C}$, free salicylic acid content; and $\mathbf{D}, M d P R 1, M d P R 5$, and $M d N P R l$ expression levels were determined. $p I R$ and $T R V$ were used as the controls. 
AtWRKY44 is able to activate SA-induced disease resistance by directly binding to the promoter of AtWRKY7O (Shim et al. 2013). In Brassica napus, BnWRKY33 can positively regulate Sclerotinia sclerotiorum, whereas BnWRKY15 binds to the $B n W R K Y 33$ promoter, inhibits the transcriptional activation of $B n W R K Y 33$, and reduces the resistance of plants to infection by $S$. sclerotiorum (Liu et al. 2018a). In rice, OsWRKY67 can bind to the promoters of PRIa and PRIO and activate their expression to enhance resistance to leaf blast, panicle blast, and bacterial blight and accumulate endogenous SA (Liu et al. 2018b). In this study, a WRKY transcription factor (MdWRKY46) interacting with the MdPBS3.1 promoter was identified (Fig. 4). In addition, experiments demonstrated that MdPBS3.1 could be activated by MdWRKY46 to enhance resistance to infection by $B$. dothidea (Figs. 5 and 6). Upon repression of MdPBS3.1 and MdWRKY46 in apple peel, the increased SA content suggested that MdWRKY46 contributes to the accumulation to SA partially in an MdPBS3.1-dependent

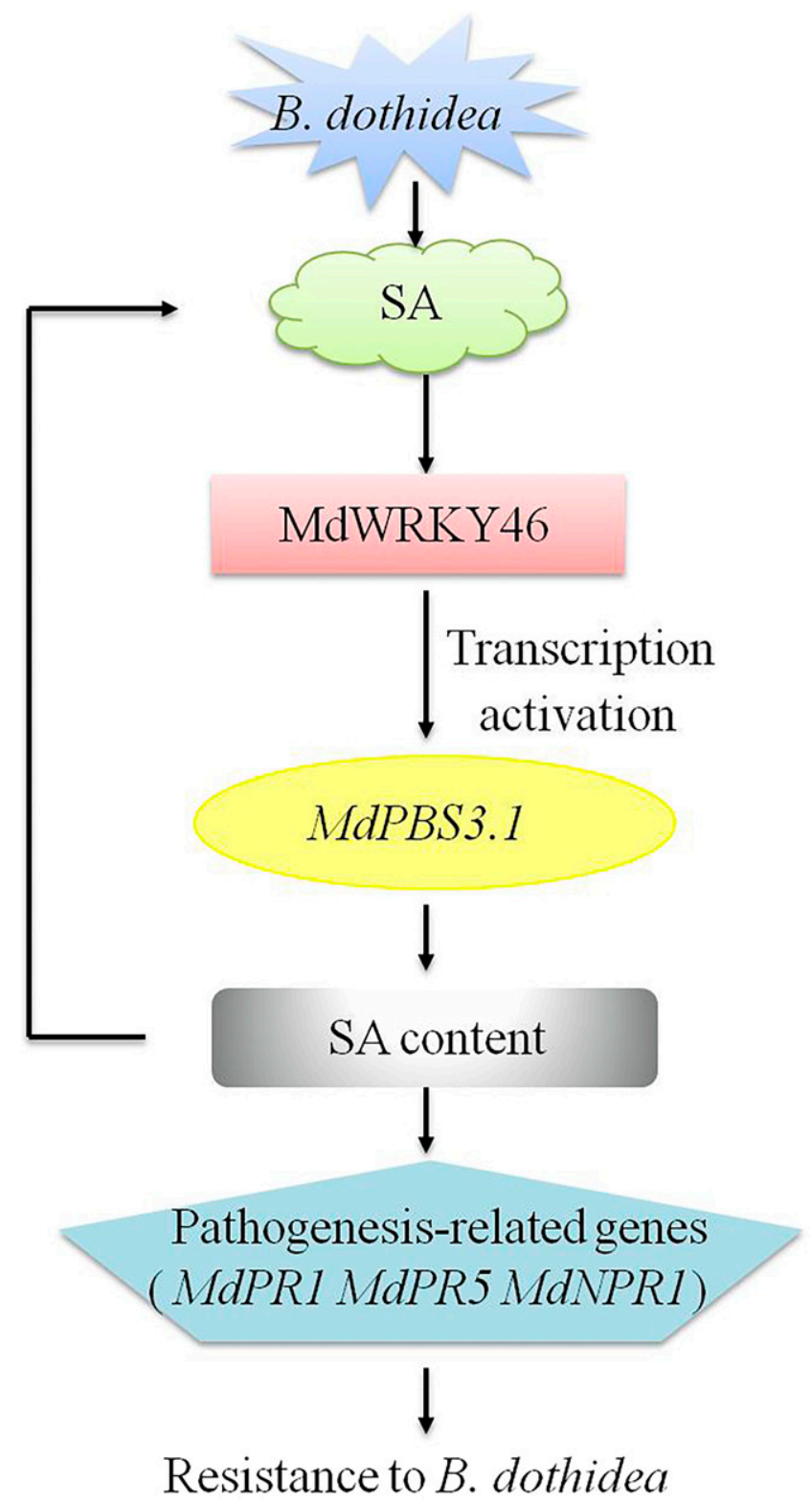

Fig. 7. Model demonstrating the regulatory mechanism of resistance to Botryosphaeria dothidea in the salicylic acid signaling pathway by MdWRKY46 through its interaction with and activation by the MdPBS3.1 promoter. way. When the W-box 2 motif was mutated, the promoter of MdPBS3.1 also responded to B. dothidea (Fig. 3), suggesting that other transcription factors may mediate the activity of MdPBS3.1 during SA signaling and disease resistance.

\section{The expression of $P R$ genes induced by MdWRKY46} in a MdPBS3.1-dependent way against $B$. dothidea.

SA-mediated plant resistance is mainly regulated by PR proteins. $P R$ genes strictly control plant growth and defense (Linthorst 1991; van Verk et al. 2008). In Arabidopsis, PRl is an SA-inducible marker gene regulated by WRKY50 and TGA transcription factors synergistically (Hussain et al. 2018). As an SA receptor, NPR1 has a indispensable function in the transcriptional regulation of plant immunity (Ding et al. 2018). When a plant is infected with a pathogen, it often accumulates SA, and the expression of disease-resistant genes for immunity increases (Zheng et al. 2015). Moreover, MdPBS3.1 and $M d W R K Y 46$ can positively regulate $B$. dothidea; positively regulate the expression of $M d P R 1, M d P R 5$, and $M d N P R 1$; and accumulate SA (Figs. 2 and 6). Thus, it is likely that MdWRKY46 can positively regulate resistance to $B$. dothidea by transcriptionally activating MdPBS3.1 through the SAmediated pathway.

\section{Conclusion.}

These results reveal that the expression of MdWRKY46 was induced by SA treatment. MdWRKY46 activated the expression of $M d P B S 3.1$ by directly binding to its promoter, resulting in the increase of MdPBS3.1 transcription. Although the overexpression of $M d P B S 3.1$ promoted the accumulation of SA, the expression of $P R$ genes, including $M d P R 1, M d P R 5$, and $M d N P R 1$, resulted in enhanced resistance to $B$. dothidea in apple. Therefore, we hypothesize that there is a positive loop in the regulation pathway of SA-MdWRKY46-MdPBS3.1-SA during biotic stress induced by agents such as $B$. dothidea (Fig. 7). In addition, there may be other mechanisms that regulate the expression of $M d P B S 3.1$, which requires further investigation.

\section{MATERIALS AND METHODS}

Plant materials and growth conditions.

Plant materials studied included bagged 'Fuji' apples (Malus domestica), 'Orin' apple calli, and 'Royal Gala' apple seedlings. 'Fuji' apples were used for apple injection assays and pathogen infection assays. 'Fuji' apples were picked 150 days after flowering and stored at $28^{\circ} \mathrm{C}$ in darkness for 4 days after infection with $B$. dothidea. 'Orin' apple calli were used for GUS staining and activity detection, genetic transformation, and pathogen infection assays. Apple calli were cultured on Murashige and Skoog (MS) medium supplemented with 2, 4dichlorophenoxyacetic acid at $0.5 \mathrm{mg} \cdot \mathrm{L}^{-1}, 6$-benzyl adenine (6BA) at $1.5 \mathrm{mg} \cdot \mathrm{L}^{-1}$, sucrose at $30 \mathrm{~g} \cdot \mathrm{L}^{-1}$, and agar at $8 \mathrm{~g} \cdot \mathrm{L}^{-1}$ at $25^{\circ} \mathrm{C}$ in darkness. 'Royal Gala' apple seedlings were used for assays that tested the response to $B$. dothidea. 'Royal Gala' apple seedlings were treated with SA at $150 \mu \mathrm{mol} \cdot \mathrm{L}^{-1}$ for $0 \mathrm{~h}, 1$ h, $3 \mathrm{~h}, 6 \mathrm{~h}, 12 \mathrm{~h}$, and $24 \mathrm{~h}$. 'Royal Gala' apple seedlings were cultured on MS medium supplemented with 6-BA at $1.0 \mathrm{mg} \cdot \mathrm{L}^{-1}$, naphthylacetic acid at $0.2 \mathrm{mg} \cdot \mathrm{L}^{-1}$, sucrose at 30 $\mathrm{g} \cdot \mathrm{L}^{-1}$, and agar at $8 \mathrm{~g} \cdot \mathrm{L}^{-1}$ at $25^{\circ} \mathrm{C}$ under $16 \mathrm{~h}$ of light.

\section{Vector construction and transformation.}

The promoter sequence of $M d P B S 3.1$ was used to construct the recombinant plasmids, including proMdPBS3.1-GUS, proMdPBS3.1-box2-pHIS2, and proMdPBS3.1-box2-pGreenII $0800-L U C$. The vector construction of the mutant promoter of MdPBS3.1 (proMdPBS3.1-GUS(m1 and m2)) was as follows: i) the recombinant proMdPBS3.1-GUS plasmid was used as the 
template; ii) two DNA fragments were amplified using primers $\mathrm{F} 1 / \mathrm{R} 1$ and $\mathrm{F} 2 / \mathrm{R} 2$ of proMdPBS3.1-GUS( $\mathrm{ml})$ or proMdPBS3.1$G U S(m 2)$, respectively; and iii) the proMdPBS3.1-GUS(m1) or proMdPBS3.1-GUS(m2) fragment was obtained using primers F1/R2 of proMdPBS3.1-GUS(m1) or proMdPBS3.1-GUS(m2) after combining the two DNA fragments, which served as the template.

Full-length MdPBS3.1 cDNA was used to generate $p I R$ $M d P B S 3.1$ and MdPBS3.1-GFP. Approximately $250 \mathrm{bp}$ of the nonconserved domain sequence of $M d P B S 3.1$ was used to construct TRV-MdPBS3.1, and the reverse complement of MdPBS3.1 was used to construct MdPBS3.1-Anti. Full-length MdWRKY46 cDNA was used to generate the recombinant plasmids, including MdWRKY46-AD, MdWRKY46-GFP, MdWRKY46-GST, MdWRKY46-pGreenII 62-SK, and pIR-MdWRKY46. All primers in this study are listed in Supplementary Table S1.

MdWRKY46-GFP, MdPBS3.1-GFP, and MdPBS3.1-Anti were transformed into apple calli by Agrobacterium tumefaciensmediated genetic transformation as previously described (An et al. 2018a). After incubation of Agrobacterium tumefaciens-containing recombinant plasmids or WT calli for $30 \mathrm{~min}$, the infected calli were cultured on 'Orin' calli medium without antibiotics in darkness for 2 days, and the infected calli were transferred to the 'Orin' calli medium containing antibiotics for screening.

\section{qRT-PCR assays.}

qRT-PCR was performed as previously described (Qi et al. 2018). MdACTIN was used as the reference gene. qRT-PCR reaction conditions were as follows: predenaturation at $95^{\circ} \mathrm{C}$ for $10 \mathrm{~min}$, followed by 40 cycles of denaturation at $95^{\circ} \mathrm{C}$ for 15 $\mathrm{s}$, annealing at $56^{\circ} \mathrm{C}$ for $15 \mathrm{~s}$, and extension at $65^{\circ} \mathrm{C}$ for $10 \mathrm{~s}$. The fluorescence signal was obtained in step 3 of each cycle. The data were analyzed using the $2^{-\Delta \Delta \mathrm{Ct}}$ method.

\section{EMSA.}

The relationship between MdWRKY46 and MdPBS3.1 was investigated using the LightShift Chemiluminescent EMSA kit (Thermo Fisher Scientific). The MdWRKY46-GST fusion protein, unlabeled probe, biotin-labeled probe, and mutant $M d P B S 3.1$ probe or WT MdPBS3.1 probe were incubated in binding buffer at $24^{\circ} \mathrm{C}$ for $20 \mathrm{~min}$. The samples were separated by polyacrylamide gel electrophoresis. The GST protein was used as a negative control.

\section{ChIP-qPCR assays.}

ChIP-PCR assays were performed using the EpiTect ChIP OneDay kit (SinaSun), according to manufacturers' instructions. The overexpression of MdWRKY46-GFP and the GFP-labeled empty vector in calli were used for ChIP-PCR assays. The GFP antibody was used in this assay. Enriched DNA fragments were detection by qPCR.

\section{Y1H assays.}

The MATCHMAKER One-Hybrid System (Clontech) was used to conduct $\mathrm{Y} 1 \mathrm{H}$ assays, according to manufacturers' instructions. The PGADT7-Rec2 yeast expression vector containing the GAL4 activation domain and the cDNA library of apple peel cells, and the bait, $p H I S 2$ reporter vector, which contained the critical positive regulatory elements of the MdPBS3.1 promoter, were cotransformed into the Y187 strain. The transformants were screened on Leu-, Trp-, and His-deficient medium supplemented with $20 \mathrm{mM}$ 3-AT for 3 to 4 days. The positive clones were extracted and transformed into Escherichia coli DH5 $\alpha$ for sequence analysis. The amino acid sequences of the positive clones were checked using the NCBI website.

MdWRKY46-AD and proMdPBS3.1-box2-pHIS2 were cotransformed to the Y187 yeast strain. The cotransformed
Y187 yeast strain was cultured on Leu-, Trp-, and His-deficient medium supplemented with $20 \mathrm{mM}$ and $80 \mathrm{mM} 3-\mathrm{AT}$.

\section{Transient expression assays.}

Transient expression assays were performed as previously described (An et al. 2018b). Agrobacterium tumefacienscontaining proMdPBS3.1-box2-pGreenII 0800-LUC and MdWRKY46-pGreenII 62-SK were resuspended in $\mathrm{MgCl}_{2}$ at $2 \mathrm{~g} \cdot \mathrm{L}^{-1}$ and MES at $2.132 \mathrm{~g} \cdot \mathrm{L}^{-1}$ and coinjected into the leaves of Nicotiana benthamiana. After 3 days, luminescence was detected using a live-cell imager.

\section{Apple injection assays.}

Apple injection assays were performed as previously described (An et al. 2018c). Briefly, 'Fuji' apples were picked 150 days after flowering, and TRV-MdPBS3.1 and $p I R$ $M d W R K Y 46$ were then mixed and coinjected into apple peels. pIR-MdWRKY46 was also injected into apple peels. After 2 days, the free SA content in the apple peels was quantified by high-performance liquid chromatography, related gene expression was measured by qRT-PCR assay, and the injection holes were inoculated with $B$. dothidea mycelia. The injected apples were maintained at $28^{\circ} \mathrm{C}$ in darkness for 4 days.

\section{Pathogen infection assays.}

Transgenic calli (MdWRKY46-GFP, MdPBS3.1-Anti, and $M d W R K Y 46-G F P / M d P B S 3.1-A n t i)$ and WT calli were grown on calli medium for 10 days. Subsequently, these calli and B. dothidea mycelia were cocultured for 4 days at $28^{\circ} \mathrm{C}$ in darkness.

B. dothidea was inoculated into 'Fuji' apples and maintained in darkness at $28^{\circ} \mathrm{C}$ for 0 days, 1 day, and 2 days. 'Fuji' apples picked at the same time were drilled and maintained in darkness at $28^{\circ} \mathrm{C}$ for 0 days, 1 day, and 2 days as a control. The inoculated peel was excised and frozen in liquid nitrogen for qRT-PCR.

As described by He et al. (2018), the samples were infected with 0.5 -cm-diameter agar discs containing uniformly dispersed $B$. dothidea mycelia.

\section{GUS staining and activity detection.}

pGreenII 62-SK and 1300-GUS, MdWRKY46-pGreenII 62SK and 1300-GUS, pGreenII 62-SK and proMdPBS3.1-box2GUS, and MdWRKY46-pGreenII 62-SK and proMdPBS3. 1-box2-GUS were transformed into calli by vacuum infiltration. Histochemical staining was carried out to measure GUS activity in the treated calli as previously described (Zhao et al. 2016). Briefly, the transgenic apple calli were added to a GUS-staining buffer and maintained at $37^{\circ} \mathrm{C}$ in darkness for 8 h. For quantitative testing of GUS activity, the transgenic apple calli were added to extraction buffer, and the protein was quantified using the RC DC Protein Assay kit (Bio-Rad Laboratories). GUS activity was quantified using a spectrofluorometer. In addition, the calli that underwent treatment with vacuum infiltration were used for protein extraction, and western blot assays were performed to measure the GUS protein level using an anti-GUS antibody (BGI).

proMdPBS3.1-box2-GUS was transformed into calli by an Agrobacterium spp.-mediated method. Subsequently, MdWRKY46GFP was transformed by vacuum infiltration into the calli described above. GUS staining and GUS activity were performed as previously described (Zhao et al. 2016).

GUS-staining assays of 'Royal Gala' apple seedlings were performed as previously described (Zhang et al. 2018). Briefly, 'Royal Gala' apple seedlings were infiltrated in liquid medium containing proMdPBS3.1-GUS (proMdPBS3.1-GUS $(\mathrm{ml})$ or proMdPBS3.1-GUS $(\mathrm{ml})$ ) under a $65 \mathrm{kPa}$ vacuum for $20 \mathrm{~min}$. 
'Royal Gala' apple seedlings infiltrated with proMdPBS3.1GUS were inoculated with a pathogenic spore (optical density at $600 \mathrm{~nm}$ of 0.6 ) under a $65 \mathrm{kPa}$ vacuum for $10 \mathrm{~min}$. After infiltration, the 'Royal Gala' apple seedlings were incubated in GUS-staining solution for $12 \mathrm{~h}$, followed by destaining with $70 \%$ ethyl alcohol.

\section{Western blot.}

Calli were combined with sodium dodecyl sulfatepolyacrylamide gel electrophoresis loading buffer, boiled, and centrifuged. After collecting the supernatant, polypropylene gel electrophoresis was performed, and the proteins were transferred to polyvinylidene difluoride membranes (Millipore) for immunoblotting with an appropriate antibody.

\section{Free SA and SAG detection assays.}

As described previously by Verberne et al. (2002), free SA and SAG extracted from apple peels (approximately $0.1 \mathrm{~g}$ of tissue) were quantified by high-performance liquid chromatography. This experiment was performed independently three times.

\section{Bioinformatics analysis.}

The conserved domain of the MdPBS3.1 protein was analyzed using the NCBI conserved-domain online software. Phylogenetic tree analysis was performed using Mega 7.0.2 software (NCBI) as previously described (Qi et al. 2019).

\section{Statistical analysis.}

Appropriate methods, using R (3.0.2) with the R Commander package, were employed to analyze three parallel experiments statistically. The mean differences in the bars were significant at the $P_{0.05}$ level, with different letters, and not significant at the $P_{0.05}$ level, with the same letters.

\section{AUTHOR-RECOMMENDED INTERNET RESOURCES}

Apple Genome and Epigenome database: https://iris.angers.inra.fr/gddh13 NCBI website: https://www.ncbi.nlm.nih.gov

\section{LITERATURE CITED}

Abreu, M. E., and Munné-Bosch, S. 2009. Salicylic acid deficiency in NahG transgenic lines and sid2 mutants increases seed yield in the annual plant Arabidopsis thaliana. J. Exp. Bot. 60:1261-1271.

An, J. P., An, X. H., Yao, J. F., Wang, X. N., You, C. X., Wang, X. F., and Hao, Y. J. 2018a. BTB protein MdBT2 inhibits anthocyanin and proanthocyanidin biosynthesis by triggering MdMYB9 degradation in apple. Tree Physiol. 38:1578-1587.

An, J. P., Li, R., Qu, F. J., You, C. X., Wang, X. F., and Hao, Y. J. 2018b. R2R3-MYB transcription factor MdMYB23 is involved in the cold tolerance and proanthocyanidin accumulation in apple. Plant J. 96: 562-577.

An, J. P., Wang, X. F., Li, Y. Y., Song, L. Q., Zhao, L. L., You, C. X., and Hao, Y. J. 2018c. EIN3-LIKE1, MYB1, and ETHYLENE RESPONSE FACTOR3 act in a regulatory loop that synergistically modulates ethylene biosynthesis and anthocyanin accumulation. Plant Physiol. 178: 808-823.

Bai, S., Dong, C., Li, B., and Dai, H. 2013. A PR-4 gene identified from Malus domestica is involved in the defense responses against Botryosphaeria dothidea. Plant Physiol. Biochem. 62:23-32.

Barman, K., Sharma, S., Kumari, P., and Siddiqui, M. W. 2016. Salicylic acid. Pages 51-68 in: Postharvest Management Approaches for Maintaining Quality of Fresh Produce. M. W. Siddiqui, J. F. Ayala Zavala, and C. A. A. Hwang, eds. Springer International Publishing, New York.

Cao, H., Glazebrook, J., Clarke, J. D., Volko, S., and Dong, X. 1997. The Arabidopsis NPR1 gene that controls systemic acquired resistance encodes a novel protein containing ankyrin repeats. Cell 88:57-63.

Chang, M., Zhao, J., Chen, H., Li, G., Chen, J., Li, M., Palmer, I. A., Song, J., Alfano, J. R., Liu, F., and Fu, Z. Q. 2019. PBS3 protects EDS1 from proteasome-mediated degradation in plant immunity. Mol. Plant 12: 678-688.
Chen, Z., Silva, H., and Klessig, D. F. 1993. Active oxygen species in the induction of plant systemic acquired resistance by salicylic acid. Science 262:1883-1886.

Cui, H., Gobbato, E., Kracher, B., Qiu, J., Bautor, J., and Parker, J. E. 2016. A core function of EDS1 with PAD4 is to protect the salicylic acid defense sector in Arabidopsis immunity. New Phytol. 213:1802-1817.

Ding, Y., Sun, T., Ao, K., Peng, Y., Zhang, Y., Li, X., and Zhang, Y. 2018. Opposite roles of salicylic acid receptors NPR1 and NPR3/NPR4 in transcriptional regulation of plant immunity. Cell 173:1454-1467.E15.

Dong, J., Chen, C., and Chen, Z. 2003. Expression profiles of the Arabidopsis WRKY gene superfamily during plant defense response. Plant Mol. Biol. 51:21-37.

Durner, J., and Klessig, D. F. 1995. Inhibition of ascorbate peroxidase by salicylic acid and 2,6-dichloroisonicotinic acid, two inducers of plan defense responses. Proc. Natl. Acad. Sci. U.S.A. 92:11312-11316.

Enyedi, A. J., and Raskin, I. 1993. Induction of UDP-glucose:salicylic acid glucosyltransferase activity in tobacco mosaic virus-inoculated tobacco (Nicotiana tabacum) leaves. Plant Physiol. 101:1375-1380.

Fu, Z. Q., and Dong, X. 2013. Systemic acquired resistance: Turning local infection into global defense. Annu. Rev. Plant Biol. 64:839-863.

Gu, Y., Zavaliev, R., and Dong, X. 2017. Membrane trafficking in plant immunity. Mol. Plant 10:1026-1034.

Hagen, G., Kleinschmidt, A., and Guilfoyle, T. 1984. Auxin-regulated gene expression in intact soybean hypocotyl and excised hypocotyl sections. Planta 162:147-153.

Hammond-Kosack, K. E., and Jones, J. D. J. 1996. Resistance genedependent plant defense responses. Plant Cell 8:1773-1791.

Hao, Q., Wang, W., Han, X., Wu, J., Lyu, B., Chen, F., Caplan, A., Li, C., Wu, J., Wang, W., Xu, Q., and Fu, D. 2018. Isochorismate-based salicylic acid biosynthesis confers basal resistance to Fusarium graminearum in barley. Mol. Plant Pathol. 19:1995-2010.

He, X., Huo, Y., Liu, X., Zhou, Q., Feng, S., Shen, X., Li, B., Wu, S., and Chen, X. 2018. Activation of disease resistance against Botryosphaeria dothidea by downregulating the expression of MdSYP121 in apple. Hortic. Res. 5:24.

Herrera-Vásquez, A., Salinas, P., and Holuigue, L. 2015. Salicylic acid and reactive oxygen species interplay in the transcriptional control of defense genes expression. Front. Plant Sci. 6:171.

Hu, Y., Dong, Q., and Yu, D. 2012. Arabidopsis WRKY46 coordinates with WRKY70 and WRKY53 in basal resistance against pathogen Pseudomonas syringae. Plant Sci. 185-186:288-297.

Hussain, R. M. F., Sheikh, A. H., Haider, I., Quareshy, M., and Linthorst, H. J. M. 2018. Arabidopsis WRKY50 and TGA transcription factors synergistically activate expression of PR1. Front. Plant Sci. 9:930.

Jayakannan, M., Bose, J., Babourina, O., Rengel, Z., and Shabala, S. 2015. Salicylic acid in plant salinity stress signalling and tolerance. Plant Growth Regul. 76:25-40.

Jia, X., Zeng, H., Wang, W., Zhang, F., and Yin, H. 2018. Chitosan oligosaccharide induces resistance to Pseudomonas syringae pv. tomato DC3000 in Arabidopsis thaliana by activating both salicylic acid- and jasmonic acid-mediated pathways. Mol. Plant-Microbe Interact. 31: 1271-1279.

Jones, J. D. G., and Dangl, J. L. 2006. The plant immune system. Nature 444:323-329.

Journot-Catalino, N., Somssich, I. E., Roby, D., and Kroj, T. 2006. The transcription factors WRKY11 and WRKY17 act as negative regulators of basal resistance in Arabidopsis thaliana. Plant Cell 18:3289-3302.

Kenton, P., Darby, R. M., Shelley, G., and Draper, J. 2000. A PR-5 gene promoter from Asparagus officinalis (AoPRT-L) is not induced by abiotic stress, but is activated around sites of pathogen challenge and by salicylate in transgenic tobacco. Mol. Plant Pathol. 1:367-378.

Kim, K. C., Fan, B., and Chen, Z. 2006. Pathogen-induced Arabidopsis WRKY7 is a transcriptional repressor and enhances plant susceptibility to Pseudomonas syringae. Plant Physiol. 142:1180-1192.

Klessig, D. F., Choi, H. W., and Dempsey, D. A. 2018. Systemic acquired resistance and salicylic acid: Past, present, and future. Mol. PlantMicrobe Interact. 31:871-888.

Kuźniak, E., Wielanek, M., Chwatko, G., Głowacki, R., Libik-Konieczny, M., Piątek, M., Gajewska, E., and Skłodowska, M. 2015. Salicylic acid and cysteine contribute to arbutin-induced alleviation of angular leaf spot disease development in cucumber. J. Plant Physiol. 181:9-13.

Kvaratskhelia, M., George, S. J., and Thorneley, R. N. 1997. Salicylic acid is a reducing substrate and not an effective inhibitor of ascorbate peroxidase. J. Biol. Chem. 272:20998-21001.

Lee, H. I., León, J., and Raskin, I. 1995. Biosynthesis and metabolism of salicylic acid. Proc. Natl. Acad. Sci. U.S.A. 92:4076-4079.

Lee, M. W., Jelenska, J., and Greenberg, J. T. 2008. Arabidopsis proteins important for modulating defense responses to Pseudomonas syringae that secrete HopW1-1. Plant J. 54:452-465. 
Lehmann, S., Serrano, M., L'Haridon, F., Tjamos, S. E., and Metraux, J. P. 2015. Reactive oxygen species and plant resistance to fungal pathogens. Phytochemistry 112:54-62.

Li, J., Brader, G., Kariola, T., and Palva, E. T. 2006. WRKY70 modulates the selection of signaling pathways in plant defense. Plant J. 46:477-491.

Li, M., Chen, H., Chen, J., Chang, M., Palmer, I. A., Gassmann, W., Liu, F., and Fu, Z. Q. 2018. TCP transcription factors interact with NPR1 and contribute redundantly to systemic acquired resistance. Front. Plant Sci. 9:1153.

Linthorst, H. J. M., and Van Loon, L. C. 1991. Pathogenesis-related proteins of plants. Crit. Rev. Plant Sci. 10:123-150.

Liu, F., Li, X., Wang, M., Wen, J., Yi, B., Shen, J., Ma, C., Fu, T., and Tu, J. 2018a. Interactions of WRKY15 and WRKY33 transcription factors and their roles in the resistance of oilseed rape to Sclerotinia infection. Plant Biotechnol. J. 16:911-925.

Liu, Q., Li, X., Yan, S., Yu, T., Yang, J., Dong, J., Zhang, S., Zhao, J., Yang, T., Mao, X., Zhu, X., and Liu, B. 2018b. OsWRKY67 positively regulates blast and bacteria blight resistance by direct activation of $P R$ genes in rice. BMC Plant Biol. 18:257.

Lu, H., Greenberg, J. T., and Holuigue, L. 2016. Editorial: Salicylic acid signaling networks. Front. Plant Sci. 7:238.

Malamy, J., Hennig, J., and Klessig, D. F. 1992. Temperature-dependent induction of salicylic acid and its conjugates during the resistance response to tobacco mosaic virus infection. Plant Cell 4:359-366.

Marsberg, A., Kemler, M., Jami, F., Nagel, J. H., Postma-Smidt, A., Naidoo, S., Wingfield, M. J., Crous, P. W., Spatafora, J. W., Hesse, C. N., Robbertse, B., and Slippers, B. 2017. Botryosphaeria dothidea: A latent pathogen of global importance to woody plant health. Mol. Plant Pathol. 18:477-488.

Mou, Z., Fan, W., and Dong, X. 2003. Inducers of plant systemic acquired resistance regulate NPR1 function through redox changes. Cell 113: 935-944.

Nawrath, C., and Métraux, J. P. 1999. Salicylic acid induction-deficient mutants of Arabidopsis express $P R-2$ and $P R-5$ and accumulate high levels of camalexin after pathogen inoculation. Plant Cell 11:1393-1404.

Nobuta, K., Okrent, R. A., Stoutemyer, M., Rodibaugh, N., Kempema, L., Wildermuth, M. C., and Innes, R. W. 2007. The GH3 acyl adenylase family member PBS3 regulates salicylic acid-dependent defense responses in Arabidopsis. Plant Physiol. 144:1144-1156.

Noctor, G., Gomez, L., Vanacker, H., and Foyer, C. H. 2002. Interactions between biosynthesis, compartmentation and transport in the control of glutathione homeostasis and signalling. J. Exp. Bot. 53:1283-1304.

Okrent, R. A., Brooks, M. D., and Wildermuth, M. C. 2009. Arabidopsis GH3.12 (PBS3) conjugates amino acids to 4-substituted benzoates and is inhibited by salicylate. J. Biol. Chem. 284:9742-9754.

Pandey, S. P., Roccaro, M., Schön, M., Logemann, E., and Somssich, I. E. 2010. Transcriptional reprogramming regulated by WRKY18 and WRKY40 facilitates powdery mildew infection of Arabidopsis. Plant J. 64:912-923.

Pandey, S. P., and Somssich, I. E. 2009. The role of WRKY transcription factors in plant immunity. Plant Physiol. 150:1648-1655.

Qi, C. H., Zhao, X. Y., Jiang, H., Liu, H. T., Wang, Y. X., Hu, D. G., and Hao, Y. J. 2018a. Molecular cloning and functional identification of an apple flagellin receptor MdFLS2 gene. J. Integr. Agric. 17:2694-2703.

Qi, C. H., Zhao, X. Y., Jiang, H., Zheng, P. F., Liu, H. T., Li, Y. Y., and Hao, Y. J. 2019. Isolation and functional identification of an apple MdCER1 gene. Plant Cell Tissue Organ Cult. 136:1-13.

Ryals, J. A., Neuenschwander, U. H., Willits, M. G., Molina, A., Steiner, H. Y., and Hunt, M. D. 1996. Systemic acquired resistance. Plant Cell 8: 1809-1819.

Sarris, P. F., Duxbury, Z., Huh, S. U., Ma, Y., Segonzac, C., Sklenar, J., Derbyshire, P., Cevik, V., Rallapalli, G., Saucet, S. B., Wirthmueller, L., Menke, F. L. H., Sohn, K. H., and Jones, J. D. G. 2015. A plant immune receptor detects pathogen effectors that target WRKY transcription factors. Cell 161:1089-1100.

Shim, J. S., Jung, C., Lee, S., Min, K., Lee, Y. W., Choi, Y., Lee, J. S., Song, J. T., Kim, J. K., and Choi, Y. D. 2013. AtMYB44 regulates WRKY70 expression and modulates antagonistic interaction between salicylic acid and jasmonic acid signaling. Plant J. 73:483-495.

Shirasu, K., Nakajima, H., Rajasekhar, V. K., Dixon, R. A., and Lamb, C. 1997. Salicylic acid potentiates an agonist-dependent gain control that amplifies pathogen signals in the activation of defense mechanisms. Plant Cell 9:261-270.
Staswick, P. E., Serban, B., Rowe, M., Tiryaki, I., Maldonado, M. T., Maldonado, M. C., and Suza, W. 2005. Characterization of an Arabidopsis enzyme family that conjugates amino acids to indole-3acetic acid. Plant Cell 17:616-627.

Staswick, P. E., Tiryaki, I., and Rowe, M. L. 2002. Jasmonate response locus JAR1 and several related Arabidopsis genes encode enzymes of the firefly luciferase superfamily that show activity on jasmonic, salicylic, and indole-3-acetic acids in an assay for adenylation. Plant Cell 14: $1405-1415$

van Verk, M. C., Pappaioannou, D., Neeleman, L., Bol, J. F., and Linthorst, H. J. M. 2008. A novel WRKY transcription factor is required for induction of $P R-1 a$ gene expression by salicylic acid and bacterial elicitors. Plant Physiol. 146:1983-1995.

Vanacker, H., Carver, T. L., and Foyer, C. H. 2000. Early $\mathrm{H}_{2} \mathrm{O}_{2}$ accumulation in mesophyll cells leads to induction of glutathione during the hyper-sensitive response in the barley-powdery mildew interaction. Plant Physiol. 123:1289-1300.

Verberne, M. C., Brouwer, N., Delbianco, F., Linthorst, H. J. M., Bol, J. F., and Verpoorte, R. 2002. Method for the extraction of the volatile compound salicylic acid from tobacco leaf material. Phytochem. Anal. 13:45-50.

Wang, C., Zhang, X., Fan, Y., Gao, Y., Zhu, Q., Zheng, C., Qin, T., Li, Y., Che, J., Zhang, M., Yang, B., Liu, Y., and Zhao, K. 2015. XA23 is an executor $\mathrm{R}$ protein and confers broad-spectrum disease resistance in rice. Mol. Plant 8:290-302.

Wang, G. F., Seabolt, S., Hamdoun, S., Ng, G., Park, J., and Lu, H. 2011. Multiple roles of WIN3 in regulating disease resistance, cell death, and flowering time in Arabidopsis. Plant Physiol. 156:1508-1519.

Wang, X. D., Bi, W. S., Jing, G., Yu, X. M., Wang, H. Y., and Liu, D. Q. 2018. Systemic acquired resistance, NPR1, and pathogenesis-related genes in wheat and barley. J. Integr. Agric. 17:2468-2477.

Wang, Y., Dang, F., Liu, Z., Wang, X., Eulgem, T., Lai, Y., Yu, L., She, J., Shi, Y., Lin, J., Chen, C., Guan, D., Qiu, A., and He, S. 2013. CaWRKY58, encoding a group I WRKY transcription factor of Capsicum annuum, negatively regulates resistance to Ralstonia solanacearum infection. Mol. Plant Pathol. 14:131-144.

Wei, W., Cui, M. Y., Hu, Y., Gao, K., Xie, Y. G., Jiang, Y., and Feng, J. Y. 2018. Ectopic expression of $F v W R K Y 42$, a WRKY transcription factor from the diploid woodland strawberry (Fragaria vesca), enhances resistance to powdery mildew, improves osmotic stress resistance, and increases abscisic acid sensitivity in Arabidopsis. Plant Sci. 275:60-74.

Westfall, C. S., Herrmann, J., Chen, Q., Wang, S., and Jez, J. M. 2010. Modulating plant hormones by enzyme action: The GH3 family of acyl acid amido synthetases. Plant Signal. Behav. 5:1607-1612.

White, R. F. 1979. Acetylsalicylic acid (aspirin) induces resistance to tobacco mosaic virus in tobacco. Virology 99:410-412.

Wildermuth, M. C., Dewdney, J., Wu, G., and Ausubel, F. M. 2001. Isochorismate synthase is required to synthesize salicylic acid for plant defence. Nature 414:562-565.

Xu, L., Zhao, H., Ruan, W., Deng, M., Wang, F., Peng, J., Luo, J., Chen, Z., and $\mathrm{Yi}, \mathrm{K}$. 2017. Abnormal inflorescence meristem 1 functions in salicylic acid biosynthesis to maintain proper reactive oxygen species levels for root meristem activity in rice. Plant Cell 29:560-574.

Yalpani, N., Silverman, P., Wilson, T. M., Kleier, D. A., and Raskin, I. 1991. Salicylic acid is a systemic signal and an inducer of pathogenesis-related proteins in virus-infected tobacco. Plant Cell 3:809-818.

Yu, Y., Shi, J., Li, X., Liu, J., Geng, Q., Shi, H., Ke, Y., and Sun, Q. 2018. Transcriptome analysis reveals the molecular mechanisms of the defense response to gray leaf spot disease in maize. BMC Genomics 19:742.

Zhang, Q., Ma, C., Zhang, Y., Gu, Z., Li, W., Duan, X., Wang, S., Hao, L. Wang, Y., Wang, S., and Li, T. 2018. A single-nucleotide polymorphism in the promoter of a hairpin RNA contributes to Alternaria alternata leaf spot resistance in apple (Malus $\times$ domestica). Plant Cell 30:1924-1942.

Zhao, Q., Ren, Y. R., Wang, Q. J., Yao, Y. X., You, C. X., and Hao, Y. J. 2016. Overexpression of MdbHLH104 gene enhances the tolerance to iron deficiency in apple. Plant Biotechnol. J. 14:1633-1645.

Zheng, X. Y., Zhou, M., Yoo, H., Pruneda-Paz, J. L., Spivey, N. W., Kay, S. A., and Dong, X. 2015. Spatial and temporal regulation of biosynthesis of the plant immune signal salicylic acid. Proc. Natl. Acad. Sci. U.S.A. 112:9166-9173.

Zhou, N., Tootle, T. L., Tsui, F., Klessig, D. F., and Glazebrook, J. 1998. $P A D 4$ functions upstream from salicylic acid to control defense responses in Arabidopsis. Plant Cell 10:1021-1030. 